\title{
Linfedema penoescrotal secundario a hidrosadenitis supurativa: a propósito de un caso, revisión de las opciones quirúrgicas
}

\author{
Penile and scrotal lymphedema associated with hidradenitis suppurativa: Case report and \\ review of surgical options
}

Yvan D. Pacheco*, Orlando García-Duque y Javier Fernández-Palacios

Servicio de Cirugía Plástica, Hospital Universitario de Gran Canaria Dr. Negrín, Las Palmas de Gran Canaria, España

\begin{abstract}
Resumen
El linfedema es producto de una alteración en el drenaje linfático, y su causa más frecuente en todo el mundo es la filariasis. En nuestro medio suele encontrarse asociada a procesos neoplásicos, inflamatorios, granulomatosos, secuelas por radioterapia, desequilibrios hidroelectrolíticos y procesos idiopáticos. Puede afectar a cualquier parte del cuerpo, incluyendo el pene y el escroto. El linfedema genital es una presentación infrecuente, que corresponde al $0.6 \%$ de los linfedemas. No obstante, causa graves limitaciones funcionales, sociales y emocionales para el paciente. Con mucha frecuencia se presentan dolor, infecciones recurrentes, disfunción sexual y deformidad estética, llegando incluso a limitar la movilidad y la deambulación. Aunque existen varias opciones de tratamiento, tanto médico como quirúrgico, no se ha encontrado el ideal para esta enfermedad. Presentamos el caso de un paciente de 43 años afecto de hidrosadenitis axilar e inguinal que padece linfedema penoescrotal grave que limita seriamente su actividad habitual. El paciente fue remitido a nuestro centro tras el fracaso del tratamiento con antibióticos (ciclos de doxiciclina y clindamicina). El tratamiento quirúrgico consistió en la resección de piel y tejido celular subcutáneo hasta fascia de Buck y cobertura con injertos de piel. El resultado final fue adecuado desde un punto de vista tanto funcional como estético.
\end{abstract}

PALABRAS CLAVE: Linfedema penoescrotal. Hidrosadenitis. Tratamiento quirúrgico.

\begin{abstract}
Lymphedema is the result of an alteration of the lymphatic drainage, and its most common worldwide cause is filariasis. In our practice usually is associated to neoplasic, inflammatory and granulomatous processes, radiotherapy, hydroelectrolytic disbalances, and idiopathic. It can affect any part of the body, including the penis and scrotum. The genital lymphedema is a rare presentation, it corresponds to $0.6 \%$ of lymphedema. However, causes serious functional, social and emotional limitations for the patient. Too often have pain, recurrent infections, sexual dysfunction, cosmetic deformity, sometimes it limits mobility and ambulation. Although there are several treatment options, both medical and surgical, it has not been found ideal for this disease. We present a 43 years old patient with penoscrotal lymphedema due to hidradenitis suppurativa, it limits his normal activity. The patient was referred to our center after unsuccessful medical treatment (doxycycline and clindamycin cycles). Surgical treatment consisted of total excision of the skin and subcutaneous tissue to Buck's fascia. Split thickness skin grafts were used to cover the defect. The result was satisfactory both functionally and aesthetically.
\end{abstract}

KEY WORDS: Lymphedema penoscrotal. Hidradenitis. Surgical treatment.

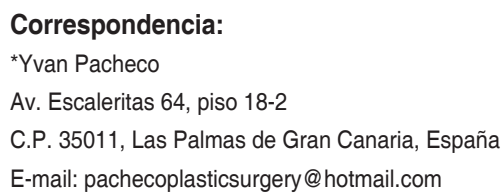

Fecha de recepción: 20-01-2017

Fecha de aceptación: 14-02-2018

DOI://dx.doi.org/10.24875/CIRU.M18000007
Cir Cir. 2018;86:84-88

Disponible en PubMed www.cirugiaycirujanos.com 


\section{Introducción}

La hidrosadenitis supurativa es una enfermedad recurrente inflamatoria de las glándulas apocrinas, que afecta a las axilas, el surco submamario, la región inguinogenital, el perineo y el surco interglúteo. Su etiología es desconocida, pero los estudios inmunohistoquímicos sugieren una causa inmunitaria mediada por las interleucinas y por el factor de necrosis tumoral alfa'.

La hidrosadenitis afecta al $1 \%$ de la población, con una incidencia de 6/100,000 personas-año $0^{1,2}$, siendo más afectadas las mujeres, pero las formas más graves se dan en los hombres ${ }^{3}$, dentro de las cuales se ha descrito el desarrollo de linfedema penoescrotal ${ }^{4}$.

El linfedema penoescrotal corresponde al $0.6 \%$ de los linfedemas ${ }^{5}$ y su causa más frecuente es la obstrucción de los canales linfáticos por el parásito de la filaria ${ }^{6}$; sin embargo, en nuestro medio esta afectación es mucho menos frecuente, siendo secundaria fundamentalmente a procesos neoplásicos, posquirúrgicos, posradioterapia y granulomatosos. Otras causas menos frecuentes son la infiltración de sustancias exógenas, como parafina o silicona, y la hidrosadenitis supurativa ${ }^{6,7}$.

Independientemente de la causa, los hallazgos histológicos son característicos. Se observan dilataciones de los capilares linfáticos rodeadas de endotelio, denominadas vesículas, que comprimen la epidermis. Estas dilataciones linfáticas confluyen en las capas subcutáneas y forman cisternas que están rodeadas de tejido muscular, cuya contracción favorece la dilatación de las vesículas superficiales ${ }^{8}$.

El tratamiento del linfedema penoescrotal debe ser acorde a su etiología; cuando el tratamiento etiológico no es posible, será quirúrgico. Las medidas compresivas y de presoterapia son poco prácticas y confortables.

Las diversas técnicas quirúrgicas se basan en la resección de piel y tejido celular subcutáneo más cobertura con colgajos cutáneos o injertos ${ }^{9}$. En los casos de linfedemas pequeños puede aplicarse la técnica de Feins ${ }^{10}$ con conservación de piel genital, y solo extirpación de tejido linfedematoso.

Cuando el linfedema es más importante, o que ocurre en la mayoría de los casos, la cobertura deberá tener en cuenta si es de pene o escroto. Para la cobertura peneana se prefieren injertos de piel total, 0 un injerto de piel parcial grueso ${ }^{10}$. Los colgajos son insatisfactorios en el pene, tanto desde el punto de vista funcional como estéticamente.
Para la cobertura del escroto se han descrito varias opciones ${ }^{9}$ :

- Colgajo de avance escrotal posterolateral (zona poco afectada en el linfedema).

- Colgajo medial de muslo.

- Injerto libre de piel parcial.

La decisión de la técnica para la cobertura testicular se tomará valorando la alteración de la termorregulación en el caso de colgajos no escrotales y la inestabilidad en el caso de injertos ${ }^{7,9}$.

\section{Caso clínico}

Presentamos el caso de un varón de 43 años con antecedente de hidrosadenitis axilar operada hace unos años, e hidrosadenitis inguinal activa, que es derivado a nuestra consulta por aumento de tamaño del pene y del escroto de meses de evolución, asociado a dolor intenso, imposibilidad funcional y sexual, y dificultad para la deambulación. Niega infiltración de sustancias exógenas y viajes al extranjero en los últimos meses. Se descartan enfermedades de transmisión sexual. En su centro de origen fue tratado con un ciclo de doxiciclina por 4 semanas y de clindamicina por 2 semanas, sin obtenerse mejoría del cuadro, por lo que fue remitido a nuestro centro.

El examen reveló un importante linfedema de pene y escroto, así como lesiones antiguas y activas de hidradenitis inguinal (Figs. 1 y 2).

Como estudios complementarios se le realizó una resonancia magnética de partes blandas que informó de grave afectación inflamatoria-infecciosa en el escroto y el pene, con microabscesos y fístulas cutáneas, y engrosamiento de partes blandas que llega a ser de $5 \mathrm{~cm}$ en el escroto y de $2.9 \mathrm{~cm}$ en el pene (Figs. 3 y 4). La ecografía abdominal y pélvica descartó procesos obstructivos. Se decide intervención conjunta con el servicio de urología.

Se realizaron desbridamiento amplio hasta la fascia de Buck en el pene y la fascia espermática externa en el escroto, fijación testicular y cobertura diferida. Se envió muestra a anatomía patológica y microbiología.

Los resultados anatomopatológicos indicaron piel con linfangiectasias, fibrosis dérmica y formación de senos delimitados por tejido inflamatorio en distintos estadios evolutivos, con formación de abscesos y reepitelización; hallazgos compatibles con hidrosadenitis supurativa. El cultivo del material purulento reveló la presencia de Prevotella bivia. 
Tras la granulación de la herida y la mejoría clínica evidente del paciente, se decide proceder a la cobertura peneana mediante un injerto de piel total tomado de la región abdominal, que se colocó de forma espiroidea para evitar la aparición de bridas retractiles, y el escroto se cubrió con injerto de piel parcial tomado del muslo izquierdo. En las revisiones se objetiva que el injerto de escroto prendió perfectamente, pero no el de pene. La cobertura definitiva de pene se logró con un injerto laminar grueso de piel parcial tomado del muslo derecho y colocado igualmente en forma espiroidea. El prendimiento fue total, siendo dado de alta a los 7 días. Tres meses después el paciente muestra estabilidad de la cobertura cutánea peneana, no presenta dolor y tiene erecciones, no se observan signos de recidiva del edema ni incurvación peneana (Figs. 5 y 6). En la revisión a los 6 meses, el paciente refiere haber iniciado la actividad sexual satisfactoria.

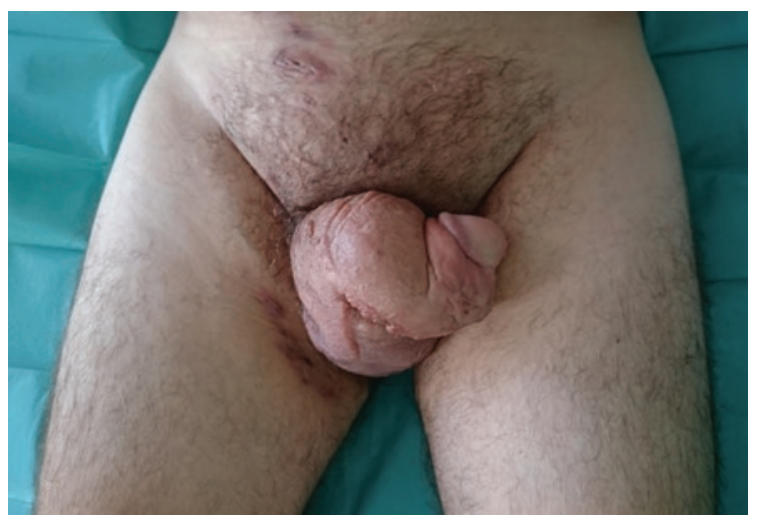

Figura 1. Varón de 43 años, con linfedema penoescrotal, de 9 meses de evolución.

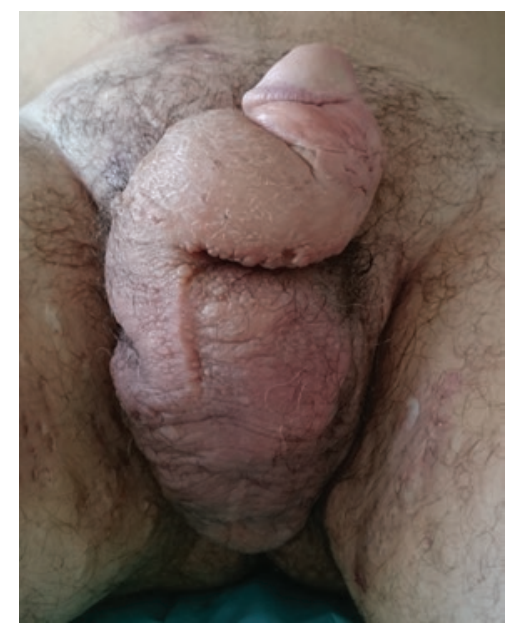

Figura 2. En el mismo paciente, nótese la deformidad adquirida, con gran compromiso funcional y estético.

\section{Discusión}

El drenaje linfático del pene y el escroto se realiza a través de los ganglios linfáticos inguinales superficiales, por lo que las enfermedades que afectan la piel y los anexos de la ingle pueden cursar con linfedema penoescrotal, como es el caso de la hidrosadenitis.

La principal causa de linfedema en el mundo es la filariasis ${ }^{6}$. En los países desarrollados se debe fundamentalmente a cirugía pélvica o radioterapia previa, y con menor frecuencia a procesos inflamatorios crónicos, como la hidrosadenitis ${ }^{6,7}$. También existen formas primarias que suelen expresarse en la pubertad, de etiología poco conocida y que suelen asociar hipoplasia de los conductos linfáticos ${ }^{11}$.

El diagnóstico de linfedema penoescrotal es clínico; las pruebas de imagen ayudan a descartar procesos

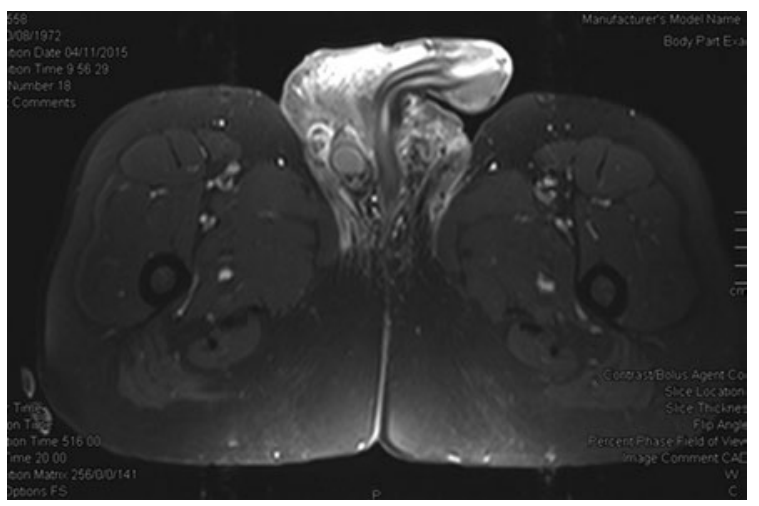

Figura 3. Resonancia magnética con contraste (corte axial) en la se aprecia el importante engrosamiento peneano, llegando en algunas zonas a $3 \mathrm{~cm}$ de diámetro.

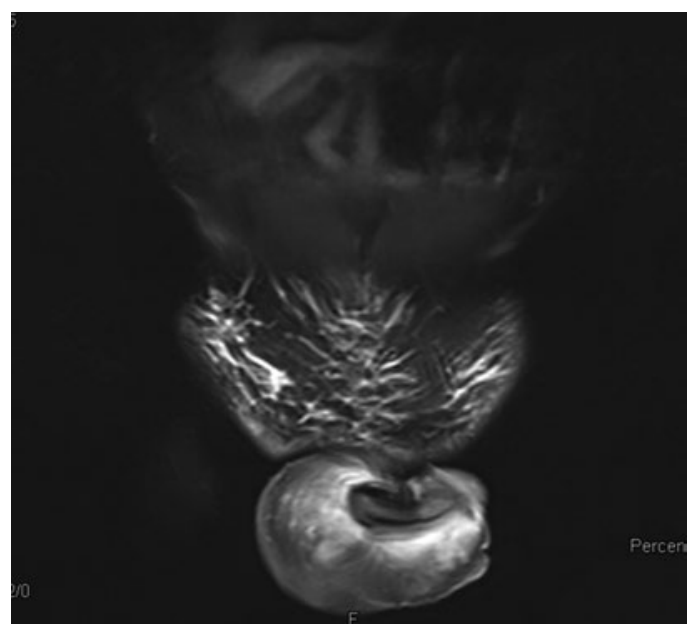

Figura 4. Resonancia magnética con contraste (corte coronal). Nótese la importante incurvación fálica del paciente. 


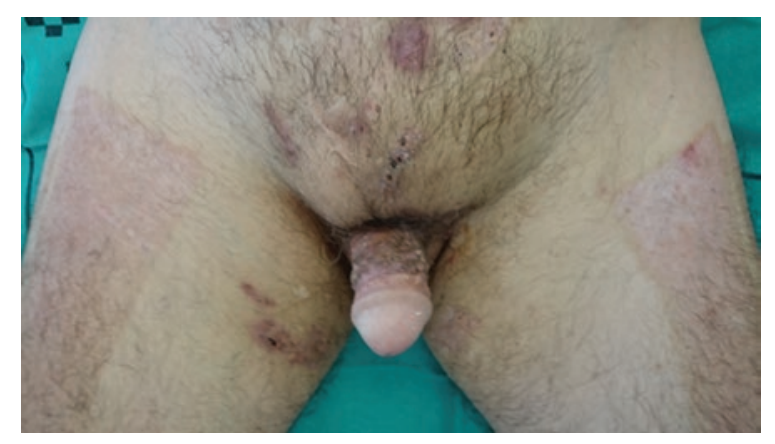

Figura 5. Posoperatorio a los 3 meses.

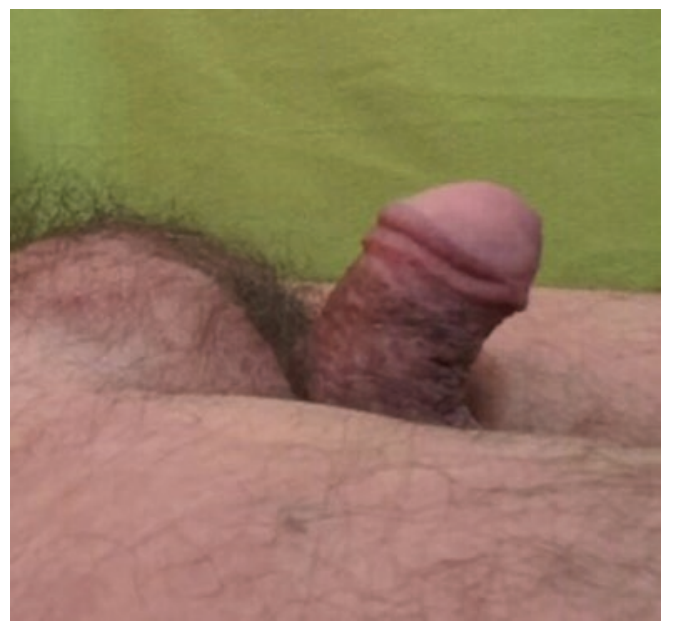

Figura 6. Cobertura cutánea estable; no se observan signos de recidiva del edema ni incurvación peneana.

obstructivos pélvicos y abdominales, y en la planificación preoperatoria ${ }^{11}$.

En algunos casos, el tratamiento puede ser etiológico y médico, aunque en la mayoría, por afectación crónica y daños irreversibles en la piel y el tejido subcutáneo, es de elección la cirugía ${ }^{9}$. La hidrosadenitis, al no tener tratamiento etiológico, es también candidata a cirugía.

Nuestro paciente llevaba una historia de casi 1 año de evolución. En su centro de origen fue tratado con antibioticoterapia en varios ciclos, sin obtener resultados. Con el transcurso del tiempo se incrementan el dolor y la deformidad, llegando a dificultar la marcha.

Los objetivos del tratamiento quirúrgico son lograr una estética aceptable y mantener la funcionalidad y la fertilidad, así como evitar las recidivas?.

El tratamiento incluye la resección completa de piel y tejido subcutáneo en el pene y el escroto. La cobertura peneana con injertos totales o parciales gruesos es aceptada casi de manera universal ${ }^{9}$, siendo la colocación de forma espiroidea la mejor en este caso para evitar bridas retráctiles. La cobertura escrotal tiene varias opciones ${ }^{10}$; se sabe que el uso de colgajos locales no escrotales puede condicionar infertilidad por alterar la termorregulación testicular, y los injertos laminares pueden ser más inestables y susceptibles ${ }^{7,9}$. Si bien los colgajos escrotales son la opción ideal, requieren indemnidad de la piel posterolateral del escroto, lo cual ocurre poco en la hidrosadenitis genital ${ }^{9,10,12}$.

En nuestro paciente, los primeros injertos de piel total en el pene no prendieron, creemos que por el grosor de la zona donante, mientras que los injertos parciales gruesos prendieron muy bien. Así se obtuvo un resultado muy satisfactorio, con gran mejora funcional, estética y social a los 3 y 6 meses de revisión.

\section{Conclusiones}

El linfedema penoescrotal secundario a hidrosadenitis supurativa es un problema poco frecuente, que provoca grandes limitaciones funcionales, estéticas y sociales en los pacientes.

Creemos que es importante reportar nuestra experiencia en el manejo de esta patología y nuestros resultados, en aras de facilitar las decisiones terapéuticas en el personal hospitalario familiarizado con esta afección.

Concluimos que, en caso de linfedema peneano crónico con importante afectación funcional, el tratamiento quirúrgico basado en la extirpación radical de piel y tejido celular subcutáneo, y su posterior cobertura con injertos cutáneos, continúa siendo una de las mejores opciones terapéuticas.

\section{Responsabilidades éticas}

Protección de personas y animales. Los autores declaran que para esta investigación no se han realizado experimentos en seres humanos ni en animales.

Confidencialidad de los datos. Los autores declaran que han seguido los protocolos de su centro de trabajo sobre la publicación de datos de pacientes.

Derecho a la privacidad y consentimiento informado. Los autores han obtenido el consentimiento informado de los pacientes y/o sujetos referidos en el artículo. Este documento obra en poder del autor de correspondencia.

\section{Financiamiento}

No se han recibido subvenciones para este trabajo.

\section{Conflicto de intereses}

No existen conflictos de intereses. 


\section{Bibliografía}

1. Jemec GB. Clinical practice. Hidradenitis suppurativa. N Engl J Med. 2012;366:158-64

2. Jemec GB, Heidenheim M, Nielsen NH. The prevalence of hidradenitis suppurativa and its potential precursor lesions. J Am Acad Dermatol. 1996;35:191-4

3. Vazquez BG, Alikhan A, Weaver AL, et al. Incidence of hidradenitis suppurativa and associated factors: a population-based study of Olmsted County, Minnesota. J Invest Dermatol. 2013;133:97-103.

4. Recalde-Losada C, Rubio-Verdú R, Solesio Pilarte F, et al. Abordaje quirúrgico de la elefantiasis escrotal, a propósito de dos casos graves. Cir Plast Iberolatinoam. 2014;40:205-12.

5. Schook C, Kulungowski A, Greene A, et al. Male genital lymphedema: clinical features and management in 25 pediatric patients. J Pediatr Surg. 2014;49:1647-51.
6. Dandapat MC, Mohapatro SK, Patro SK. Elephantiasis of the penis and scrotum. A review of 350 cases. Am J Surg. 1985;149:686-90.

7. García-Tutor E. Tratamiento quirúrgico del linfedema peneano secundario a hidrosadenitis supurativa. Actas Urol Esp. 2005;29:519-22.

8. Chéliz G, Belinki J, Kogan D, et al. Linfedema crónico genital. Plástica reconstructiva. Rev Arg Urol. 2004;69:181-3.

9. McDougal WS. Lymphedema of the external genitalia. J Urol. 2003; 170:711-6.

10. Feins NR. A new surgical technique for lymphedema of the penis and scrotum. J Pediatr Surg. 1980;15:787.

11. Halperin TJ, Slavin SA, Olumi AF, et al. Surgical management of scrotal lymphedema using local flaps. Ann Plast Surg. 2007;59:67-72.

12. Malloy TR, Wein AJ, Gross P. Scrotal and penile lymphedema: surgical considerations and management. J Urol. 1983;130:263-5. 